\title{
A Implantação do SUS e o processo de descentralização na área da saúde até
} 2002.

\author{
Claudia Valentina de Arruda Campos' ${ }^{1}$ \\ Ana Maria Malik2 ${ }^{2}$ \\ Ricardo Luiz Pereira Bueno3 ${ }^{3}$
}

RESUMO: Este texto estuda sucintamente o início da implementação do SUS, desde a 8a Conferência Nacional de Saúde até a edição da NOAS 02/02, com ênfase nos movimentos de descentralização apontados pela legislação e apresentando os programas prioritários para fins de financiamento em saúde.

PALAVRAS-CHAVE: Sistema Único de Saúde (SUS); Políticas de Saúde; Políticas Públicas; Descentralização.

Title: SUS implementation and the decentralization in the health field until 2002

SUMMARY: This paper studies in a summarized way the beginning of SUS implementation, starting at the 8th National Health Conference and arriving at NOAS

02/02. It emphasizes decentralization according to health regulation and health priorities, in terms of health financing.

KEY WORDS: Unified Health System (SUS); Health Policies; Public Policies;

Decentralization.

\footnotetext{
${ }^{1}$ Mestre em Administração Pública e Governo pela FGV-EAESP, consultora em RH. claudiavalentina@gvmail.br

${ }^{2}$ Professora da FGV-EAESP. Av. 9 de Julho 2029. $5^{\circ}$ andar. CEP 01313-902, SP- SP. Brasil. anamalik@fgvsp.br

${ }^{3}$ Mestre em Administração Pública e Governo pela FGV-EAESP, doutorando em Administração pela UFRGS, consultor em

Economia da Saúde da Johnson \& Johnson Produtos Profissionais, ricardolpb@uol.com.br
} 


\section{Apresentação}

O presente texto visa a circunstanciar o momento pelo qual passava o SUS (Sistema Único de Saúde) quando foram encaminhados projetos realizados na área de saúde pelos governos subnacionais para o programa Gestão Pública e Cidadania da FGV-EAESP (GPC). Tendo em vista que foram estudados projetos desenhados entre 1996 e 2002, este é o período aqui discutido. Os textos utilizados para a realização desta análise foram, além da legislação específica (as NOBs e as NOASs), publicações do Ministério da Saúde, artigos de pesquisadores da área de saúde e de gestão pública e informações contidas nos sites do Ministério da Saúde, do Conselho Nacional das Secretarias Estaduais de Saúde (CONASS), do Conselho Nacional das Secretarias Municipais de Saúde (CONASEMS), do Conselho Nacional de Saúde, da Fundação Nacional de Saúde, (FUNASA) da Agência Nacional de Saúde (ANS) e do Diário Oficial da União (DOU).

Os critérios utilizados pelos autores para identificar programas potencialmente prioritários ou incentivados pelo Ministério junto aos Estados e Municípios (e transformados em projetos posteriormente apresentados ao GPC) foram a menção desses programas nos documentos oficiais acima citados e a explicitação de incentivos a serem oferecidos quando da implantação dos programas.

\section{A criação do SUS e suas normas operacionais básicas}

O Sistema Único de Saúde (SUS) resultou do movimento da Reforma Sanitária no Brasil. Na 8a Conferência Nacional de Saúde, realizada em 1986, foram estabelecidos seu modelo e suas grandes diretrizes. Ao final da Conferência foi formada a Comissão Nacional de Reforma Sanitária, responsável pela elaboração do capítulo de saúde na Constituição, a partir do qual foi elaborada a lei 8080 de 1990.

Na Constituição Federal de 1988, onde a saúde foi colocada como um direito social e a seguridade social como ação para garantir o direito à saúde, o SUS acabou incluído como um dos componentes do orçamento da Seguridade Social. Os princípios estabelecidos para o SUS, constantes na Constituição são: descentralização, integração, regionalização, hierarquização e universalização dos serviços. Estes devem ser gerenciados por meio de 
A IMPLANTAÇÃO DO SUS E O PROCESSO DE DESCENTRALIZAÇÃO NA ÁREA DA SAÚDE ATÉ 2002

comando único por nível de governo e estar submetidos ao controle social. Os princípios que regem o SUS são a universalidade, a eqüidade e a integralidade da assistência.

A legislação por meio da qual se previu a implementação do sistema delimitou atribuições por nível de governo. Ao governo federal caberia o papel de formular e conduzir a Política Nacional de Saúde; aos Estados caberiam funções de gestão, controle, coordenação e elaboração, além da prestação de serviços de maior complexidade e custo, de forma coordenada com os outros níveis e aos municípios caberia planejar, gerir e coordenar o sistema de saúde em sua base territorial, assim como executar os serviços voltados para a atenção básica (ARRETCHE \& MARQUES, 2002). Desde as primeiras discussões realizadas no âmbito do chamado partido sanitário com vistas à criação do SUS era previsto que a execução dos serviços de saúde deveria ser realizada no âmbito local, ou seja, pelos municípios4 o que vinha no mesmo sentido que a reforma tributária. O termo constitucional aponta para um sistema descentralizado, que pode ou não ser entendido como municipalizado. A base do modelo do SUS se encontra na separação entre a provisão de serviços e seu financiamento. A provisão deveria ficar a cargo dos municípios; o financiamento, das três esferas de governo. (ARRETCHE, 2002)

Após a definição constitucional da política de saúde foi necessária, para sua implementação, a criação de uma série de instrumentos que possibilitasse a consecução de seus objetivos. A regulamentação do SUS foi estabelecida no final de 1990, com as Leis 8.080 e 8.142. Estas leis estabeleceram alguns dos princípios operacionais do sistema, como o controle social, a descentralização e a regionalização da assistência. No âmbito do poder executivo foram criados atos normativos e administrativos voltados para a implantação e operacionalização da política de saúde (COSTA, 2003).

Em 1990 foi elaborada a primeira Norma Operacional Básica do SUS, conhecida como NOB 01/91. Esta NOB foi criticada por prestadores, além de por aqueles que defendem que saúde é muito mais do que assistência, em função de ter mudado o sistema de financiamento da área da saúde e passado a remunerar todos os prestadores, públicos e privados, apenas por produção. A crítica a este modelo deveu-se ao fato de a remuneração por procedimento estimular a realização de atos médicos e, conseqüentemente, acentuar as desigualdades regionais.

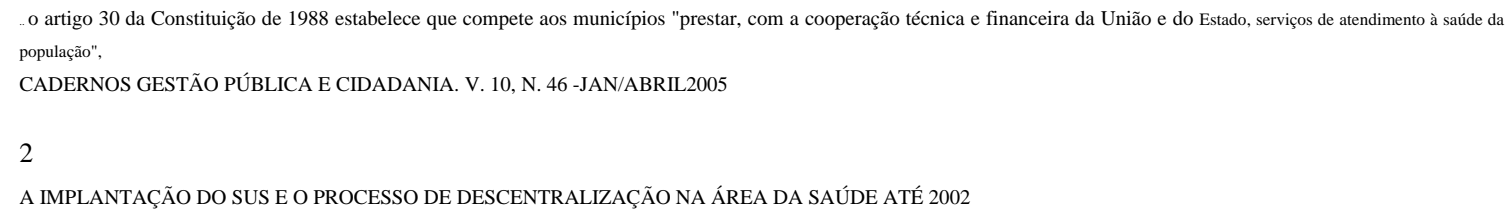

Em 1992, foi editada nova NOB, a NOB 01/92 com algumas reformulações institucionais, em particular a ampliação dos poderes das Secretarias Municipais de Saúde e, em função disso, a 
revisão dos papéis das Secretarias Estaduais e do Ministério da Saúde.

Com a crise do governo, a área de saúde também foi afetada. A 98 Conferência Nacional de Saúde, realizada nesse ano, com dois anos de atraso, teve como tema central "SaÚde: a Municipalização é o Caminho". Esta conferência destacou a necessidade da aceleração do processo de descentralização na gestão dos serviços e ações de saúde. Após sua realização, foi publicado o documento "A Ousadia de Cumprir e Fazer Cumprir a Lei", acompanhando a Norma Operacional SUS 01/93, ou NOB 01/93.

A NOB 01/93 procurou sistematizar o processo de descentralização da gestão do sistema e dos serviços por meio da proposta de um processo de transição, estabelecendo diferentes níveis de responsabilidades entre Estados, Municípios e União. Foram definidas, para os Municípios, três condições de gestão: Incipiente5, Parcial6e Semi-Plena7, em função de sua capacidade administrativa. Para os Estados foram estabeleci das duas condições de gestão: Parcial8 e SemiPlena9.

Para coordenar, gerenciar e controlar este processo foram criadas as Comissões Intergestoras Bipartite - cm (nos estados) e a Comissão Intergestora Tripartite - CIT (na instância federal), como foros permanentes de negociação e deliberação entre os diversos níveis de governo. A CIT conta com representação paritária do Ministério da Saúde, do CONASS (Conselho Nacional de Secretários Estaduais de Saúde) e CONASEMS (Conselho Nacional de Secretários Municipais de Saúde). As CIB contam com representação paritária da Secretaria Estadual de Saúde e da entidade de representação dos Municípios do Estado a que pertencem. Nestes fóruns deveriam ser pactuadas e

5 Na forma Incjpiente, cabe ao gestor municipal programar e autorizar a utilização de AIHs e dos procedimentos ambulatoriais; controlar e avaJiar os serviços ambulatoriais e hospitalares púbJicos e privados; incorporar ações básicas de saúde, nutrição. educação, vigilância epidemiológica e sanitária e desenvolver ações de vigilância. assistência e reabilitação de saúde do trabalhador. (NOB 01/93).

${ }_{6}$ Na forma Parcial de Gestão, cabe ao gestor, além de realizar o proposto na forma Incipiente, gerenciar as unidades ambulatoriais públicas do município. (NOB 01/93).

7 A Gestão Semi-Plena é a forma mais avançada de gestão proposta pela NOB 01/93, cabendo ao município a gestão de toda a rede

pública de saúde municipaL incluindo o setor privado. O município passa a receber todo o recurso para gerir o sistema. (NOB 01/93).

8 Quanto à habiJitação dos Estados, em Gestão Parcial o Estado assume as seguintes responsabilidades: programar as ações de saúde de forma integrada com os municípios; cadastrar, programar e distribuir as cotas ambulatoriais e hospitalares; controlar e avaliar a rede de serviços públicos e privados; coordenar a rede de referência estadual e o sistema de atendimento à alta complexidade (NOB 01/93). O Estado deveria receber do Ministério da Saúde, mensalmente, um valor entre o teto de recursos estabelecido para o atendimento hospitalar e ambulatorial estadual e aquilo que tivesse sido efetivamente produzido. Também foi definido um sistema de financiamento como "fator de apoio à descentralização".

" Na Gestão Semi-Plena, o Estado passa a assumir totalmente a gestão dos serviços de saúde presentes no Estado, respeitando os municípios habilitados em alguma das formas de gestão propostas pela NOB 01/93. Soma-se às atribuições estabelecidas para a gestão parcial, planejar, cadastrar, controlar e pagar os prestadores da rede pública e privada.(NOB 01/93). Para o financiamento da rede. o Estado receberia do MS o valor correspondente ao teto financeiro dos procedimentos ambulatoriais e hospitalares das redes púbJica e privada existentes. O repasse referente ao apoio à descentralização também seria recebido diretamente.

CADERNOS GESTÃO PÚBLICA E CIDADANIA, V. 10. N.46 - JAN/ ABRIL 2005

A IMPLANTAÇÃO DO SUS E O PROCESSO DE DESCENTRALIZAÇÃO NA ÁREA DA SAÚDE ATÉ 2002

3

programadas as atividades de saúde e definidos os tetos dos recursos financeiros a serem 
repassados para os sistemas de saúde Municipais e Estaduais, conforme a NOB 01103.

O processo de implantação da NOB 01/93 foi iniciado no final do ano 1994. No período de 1994 a 1996, puderam ser observadas mudanças no perfil da rede. Estas mudanças foram tanto de natureza quantitativa quanto qualitativa. Verificou-se aumento da complexidade, expansão de cobertura e considerável incremento da produção ambulatorial. (ARRETCHE \& MARQUES, 2002)10. Com as novas regras de transferência de recursos federais da NOB 01103, cerca de 66\% dos municípios do país obtiveram acréscimo de recursos na área da saúde.

A quarta Norma Operacional Básica do SUS, NOB 01196, foi implantada em 1998. Ela complementou a anterior e redefiniu critérios de habilitação. O município continuou a ser o principal operador da rede de serviços e o número de modalidades de gestão para os municípios passou de três, para dois: Gestão Plena da Atenção Básica 11 e Gestão Plena do Sistema Municipal12.

Em 1998, o Ministério da Saúde lançou o Manual para a Organização da Atenção Básica no Sistema Único de Saúde. Segundo este Manual, a Atenção Básica podia ser entendida como um conjunto de ações individuais ou coletivas no primeiro grau de atenção dos sistemas de saúde, para promoção da saúde, prevenção de agravos, tratamento e recuperação. O Manual para a Organização da Atenção Básica definia as responsabilidades na gestão da atenção básica, com um conjunto de ações: um dirigido a toda a população, e outro voltado para grupos específicos.

As ações de saúde previstas pelo Manual de Atenção Básica, a serem desenvolvidos junto a toda população, eram:

Jf) De 1982 a 1992 houve grande ampliação no número de estabelecimentos municipais de saúde, passando de 2.961 para 18.662 . Do total de estabelecimentos de saúde públicos no país, os estabelecimentos municipais passaram de 22\% para 69\%.

li Na Gestão Plena da Atenção Básica cabe ao gestor municipal elaborar a programação municipal dos serviços básicos; gerenciar todas unidades ambulatoriais existentes no município; prestar os serviços relacionados aos procedimentos cobertos pelo PAB; contratar, controlar, auditar e pagar aos prestadores dos serviços contidos no P AB; autorizar internações hospitalares e procedimentos ambulatoriais especializados pagos por produção de serviços; avaliar o impacto das ações do sistema; executar ações básicas de vigilãncia sanitária e executar ações básicas de vigilãncia epidemiológica. (NOB 01/96).

12 Na Gestão Plena do Sistema Municipal cabe ao gestor municipal elaborar toda a programação municipal, incluindo a referência ambulatorial especializada e hospitalar; gerenciar unidades próprias. ambulatoriais e hospitalares, inclusive as de referência; gerenciar as unidades ambulatoriais e hospitalares após negociação na eIB; reorganizar as unidades sob gestão pública (estatais, conveniadas e contratadas); garantir da prestação de serviços em seu território, inclusive os serviços de referência aos não-residentes, conforme PPl; contratar, controlar, auditar e pagar aos prestadores de serviços ambulatoriais e hospitalares no município; administrar a oferta de procedimentos ambulatoriais de alto custo e procedimentos hospitalares de alta complexidade conforme a PPl; avaliar o impacto de suas ações; executar as ações básicas, de média e alta complexidade em vigilãncia sanitária e executar ações de epidemiologia. (NOB 01/96).

CADERNOS GESTÃO PÚBLICA E CIDADANIA, V. 10, N. 46 - JAN/ABRIL 2005

4 A IMPLANTAÇÃO DO SUS E O PROCESSO DE DESCENTRALIZAÇÃO NA ÁREA DA SAÚDE ATÉ 2002

- Saúde educativa com foco em grupos de risco comportamentais, ambientais e alimentares; 
- Investigação de casos de doenças de notificação compulsória;

- Controle de qualidade de produtos e serviços (especialmente alimentos e serviços de saúde);

- Qualidade da água e atendimento a pequenas urgências.

As ações voltadas para grupos específicos eram as seguintes:

- Incentivo ao. aleitamento materno;

- Combate a doenças imunopreveníveis;

- Combate às carências nutricionais;

- Controle das infecções respiratórias agudas;

- Controle de doenças diarréicas;

- Controle do desenvolvimento e crescimento;

- Higiene bucal;

- Procedimentos cirúrgicos;

- Controle do pré-natal e puerpério;

- Controle do câncer cérvico-uterino e de mama;

- Planejamento familiar;

- Assistência básica às vítimas de acidentes de trabalho;

- Controle e tratamento de hanseníase e tuberculose;

- Controle e tratamento de hipertensão arterial e diabete mellitus;

- Controle da obesidade;

- Prevenção de acidentes por quedas e incentivo a grupos de auto-ajuda.

Para financiar os procedimentos e ações da Atenção Básica, a NOB 01/96 propôs uma importante mudança na forma de financiamento do sistema, com a criação do Piso da Atenção Básica (PAB), cuja vigência, associada a repasses financeiros, iniciou-se em CADERNOS GESTÃO PÚBLICA E CIDADANIA, V. ]0, N. 46 -JAN/ABRIL2005

A IMPLANTAÇÃO DO SUS E O PROCESSO DE DESCENTRALIZAÇÃO NA ÁREA DA SAÚDE ATÉ 2002

5

fevereiro de 1998. O P AB é uma forma de transferência de recursos, regular e automática, em que a verba federal é repassada diretamente aos municípios, por meio de um mecanismo 
chamado de "fundo a fundo", ou seja, do Fundo Nacional de Saúde aos fundos dos outros níveis do sistema.

A importância do mecanismo do P AB é que este dissociou o repasse financeiro do faturamento por produção, que prejudicava os municípios mais pobres onde não havia unidades de saúde. O P AB pode ser considerado como um agente intermediário no processo de descentralização do Sistema de Saúde, tendo significado um importante passo no sentido do aumento da eqüidade do sistema. (NEGRI, 2002).

O PAB tinha dois componentes: PAB fixo e PAB variável. O PAB fixo é definido pela multiplicação de um valor per capita pela população de cada município. Sua forma de repasse é direta ao município, via fundo municipal de saúde. A transferência do PAB aos Estados só ocorreria quando o município não estivesse habilitado a nenhuma das condições de gestão da NOB 01/96.

O PAB variável foi criado como estratégia de direcionamento à implantação de programas considerados estratégicos pelo Ministério da Saúde. Cabe ressaltar que, enquanto o PAB fixo deveria ser repassado com base em um valor per capita, o PAB variável seria repassado na medida em que o município aderisse aos programas prioritários definidos pelo Ministério da Saúde. O objetivo foi incentivar determinados programas [Programa de Agentes Comunitários de Saúde (PACS), Programa de Saúde da Família (PSF), Programa de Controle das Carências Nutritivas (PCCN), Programa de Controle de Endemias (PCE) e ações estratégicas do Ministério da Saúde (Assistência Farmacêutica Básica e Ações Básicas de Vigilância Sanitária, sendo que outros programas poderiam ser adicionados).

Os municípios habilitados na forma de gestão Plena da Atenção Básica passaram a receber os repasses do PAB fixo, do PAB variável e os recursos correspondentes ao Piso Básico de Vigilância Sanitária.

Já os municípios em Gestão Plena do Sistema Municipal, passaram a receber a totalidade dos recursos referentes ao Teto Financeiro da Assistência (TFA) tendo, subordinadas a eles, quase como regra geral, todas as unidades ambulatoriais especializadas e hospitalares, estatais ou privadas, estabelecidas no território municipal. Havia municípios, porém, como São Paulo, que até 2005 contavam com um grande número de hospitais subordinados à Secretaria de Estado da Saúde, à Universidade e até ao Ministério da Educação. Da mesma

CADERNOS GESTÃO PÚBLICA E CIDADANIA. V. 10. N. 46 - JANfABRIL 2005

6

A IMPLANTAÇÃO DO SUS E O PROCESSO DE DESCENTRALIZAÇÃO NA ÁREA DA SAÚDE ATÉ 2002

forma, no Rio de Janeiro, neste mesmo ano também havia hospitais subordinados ao Ministério 
da Saúde, embora se espere do município em gestão plena algum grau de comando sobre a gestão, se não sobre a gerência, dos serviços.

Quanto aos Estados, as condições de habilitação aprovadas pela NOB 96 foram: Gestão Avançada do Sistema Estadual13 e Gestão Plena do Sistema Estadual. 14

A NOB 01/96 inovou no que se refere às funções a serem desempenhadas pelos municípios e ampliou as atribuições dos Estados, cuja principal função passou a ser favorecer o processo de municipalização (ABRUCIO, 1998). Numa reflexão sobre a política de atenção básica à saúde em diferentes áreas geográficas de um país tão desigual, o PAB representou uma iniciativa orientada para a redução da desigualdade existente entre os municípios, na medida em que rompeu com a lógica dominante de alocação de recursos, o pagamento por procedimentos, pelo menos para a atenção básica.

O que se verificou, de modo geral, foi a dificuldade das Secretarias Estaduais no cumprimento de suas funções de coordenação, articulação, apoio técnico e regulação. Os Estados foram as unidades da federação que sofreram maior impacto com a redefinição de papéis estabelecida no processo de descentralização da saúde, deixando de ser o executor de ações e contratador de serviços privados e filantrópicos, e passando a exercer um papel de coordenação, apoio e regulação, para o qual não se encontravam preparados.

Para o Ministério da Saúde, a NOB 01/96 estabeleceu quatro papéis básicos: exercer a gestão do SUS no âmbito nacional; promover condições e incentivar o gestor estadual, com vistas ao desenvolvimento dos sistemas municipais; fomentar a harmonização, a integração e a' modernização dos sistemas estaduais; e elaborar as normas do SUS no âmbito nacional.

O crescimento do número de municípios habilitados ao SUS foi muito grande. Em dezembro do ano 2000, cerca de 99\% dos municípios brasileiros estavam habilitados em alguma forma de gestão junto ao SUS. Constatou-se que até o final desse ano, 582

\footnotetext{
13 Na Gestão Avançada do Sistema Estadual ficou estabelecido que cabe ao gestor estadual apresentar a Programação Pactuada Integrada (PPI) do Estado. tanto ambulatorial quanto hospitalar, com as referências intermunicipais; dispor de $60 \%$ dos municípios do Estado habilitados nas condições de gestão estabelecidas pela NOB, independente do seu contingente populacional, ou de $40 \%$ dos municípios habilitados, desde que nestes residissem $60 \%$ população, e dispor de $30 \%$ do valor do Teto Financeiro da Assistência (TFA) comprometido com transferências regulares e automáticas aos municípios.

1" Na Gestão Plena do Sistema Estadual cabe ao gestor comprovar a implementação da Programação Integrada (PPI); comprovar a operacionalização de mecanismos de controle da prestação de serviços ambulatoriais e hospitalares; dispor de $80 \%$ dos municípios habilitados nas condições de gestão estabelecidas pela NOB 01/96 independente do seu contingente populacional. ou 50\% dos municípios. desde que nestes residam 80\% da população, e dispor de 50\% do valor do Teto Financeiro da Assistência (TFA) do Estado comprometido com transferências regulares e automáticas aos Municípios. CADERNOS GESTÃO PUBLICA E CIDADANIA, V. 10, N. 46 - JAN/ABRIL 2005

A IMPLANTAÇÃO DO SUS E O PROCESSO DE DESCENTRALIZAÇÃO NA ÁREA DA SAÚDE ATÉ 2002

7

municípios que não recebiam recursos para a atenção básica antes da existência do $\mathrm{P} A B$,
} 
passaram a recebê-Ios.

A tabela abaixo apresenta um indicador do processo de descentralização do SUS: o número de municípios e Estados que receberam recursos fundo a fundo, de 1997 a 2001.

\begin{tabular}{|l|c|c|c|c|c|}
\hline \multicolumn{6}{|c|}{ Tabela I - Municípios e estados recebendo recursos fundo a fundo, de 1997 a 2001} \\
\hline Número & Dez/1997 & Dez/1998 & Dez/1999 & Dez/2000 & Dez/2001 \\
\hline Municípios & 144 & 5.049 & 5.350 & 5.450 & 5.516 \\
\hline Estados & - & 2 & 7 & 8 & 12 \\
\hline
\end{tabular}

Fonte: Secretaria de Assistência à Saúde. Relatório de Gestão 1998/2001. (SANTOS 2002:401)

Na tabela acima podemos observar o grande salto no número de municípios habilitados em alguma das formas de gestão, que passaram a receber recursos fundo a fundo de 1997 para 1998. Pode-se observar como o processo de adesão dos Estados foi mais gradual, tendo sido iniciado em 1998. Com a adesão, a capacidade federal de coordenar as ações dos governos estaduais e municipais aumentou exponencialmente.

Pelo menos três fatores podem ser considerados como tendo contribuído para o processo de descentralização do sistema de saúde no país: a existência de alto grau de consenso quanto à agenda de reformas, a estrutura de incentivos voltados à descentralização, com possibilidade de adesão ao SUS com diferentes custos e graus de responsabilidade e a redução da incerteza quanto ao fluxo de transferências de recursos.

3. Um segundo momento: as normas operacionais de assistência à saúde

Pode-se considerar que o processo de descentralização orientado pela NOB 01/96 foi bem sucedido em diversos aspectos. Por outro lado, a implementação da NOB 01/96 também permitiu que fossem identificadas suas fragilidades, particularmente no que se refere às questões intermunicipais. Ocorreram dificuldades no estabelecimento de fluxos de referência e contrareferência, de hierarquia e de integração dos serviços de saúde. Em

CADERNOS GESTÃO PÚBLICA E CIDADANIA. V. 10. N. 46 - JAN/ABRIL 2005

8 A IMPLANTAÇÃO DO SUS E O PROCESSO DE DESCENTRALIZAÇÃO NA ÁREA DA SAÚDE ATÉ 2002

busca de solucionar estes problemas, e consolidar o processo de descentralização, foi elaborada a primeira Norma Operacional da Assistência à Saúde, a NOAS 01/01.

A NOAS 01/01 foi aprovada em janeiro de 2001. Seu principal objetivo foi o fortalecimento da regionalização da assistência. Para isso foram criados três grupos de estratégias: a definição do processo de regionalização da assistência por meio da elaboração de um Plano Diretor de Regionalização (PDR); a criação de mecanismos de fortalecimento da capacidade de gestão do SUS e a atualização dos critérios de habilitação de Estados e municípios. A NQAS 01/01 inovou 
ao definir também critérios de "desabilitação" para Estados e municípios.

Nos municípios, a NOAS 01/01 ampliou a responsabilidade pela Atenção Básica, aumentando o número de ações de saúde incluído no $\mathrm{P}$ AB fixo, criando o $\mathrm{P} A B$ ampliado (PAB-A). Diferentemente da NOB 01/96, a NOAS 01/01 define os programas a serem prioritariamente atendidos por meio da atenção básica ampliada. Estes programas são: controle da tuberculose, eliminação da hanseníase, controle da hipertensão arterial, controle do diabetes mellitus, saúde da criança, saúde da mulher e saúde bucal (SOUZA, 2002).

O financiamento para estes programas encontra-se incluído no valor do $\mathrm{P}$ AB ampliado, ou PAB-A. Apesar de todos estes programas já se encontrarem entre aqueles definidos como prioritários no Manual de Atenção Básica, a NOAS 01/01 lhes deu maior destaque. Para receber o novo valor do PAB-A, os municípios tinham de se habilitar em uma das duas modalidades propostas pela NOAS 01/01: Gestão Básica Ampliada e Gestão Plena do Sistema Municipal.

Um segundo ponto da NOAS 01/01, que introduziu importante mudança em relação à NOB 01/96, apareceu na proposta de regionalização do sistema, em que os municípios passavam a ser agrupados em microrregiões de saúde. Este agrupamento devia ser proposto pela Secretaria Estadual de Saúde. Cada microrregião seria composta por um ou mais módulos de saúde. Em cada módulo, haveria um município-sede, responsável por oferecer atendimentos de média complexidade a todos os municípios do módulo. O município-sede deveria estar habilitado em Gestão Plena do Sistema e passaria a receber recursos específicos para atender a todos os moradores do módulo. (BONASSA \& CAMPOS, 2001). Os atendimentos de média complexidade, não incluídos na NOAS 01/01, assim como os procedimentos de alta complexidade, passaram a. ser referendados,

CADERNOS GESTÃO PÚBLICA E CIDADANIA. V. 10. N. 46 -JAN/ABRIL 2005

A IMPLANTAÇÃO DO SUS E O PROCESSO DE DESCENTRALIZAÇÃO NA ÁREA DA SAÚDE ATÉ 2002

9

ou seja, deveriam ser realizados em local definido, de preferência, localizado próximo ao local de residência do usuário.

Segundo a NOAS 01/01, a microrregião poderia ser "qualificada". Para isso, era necessário que o município-sede da microrregião estivesse habilitado em Gestão Plena do Sistema. Todos os demais, deveriam estar habilitados em Gestão Básica Ampliada. Após a qualificação da microrregião, os recursos fundo a fundo referentes aos procedimentos de média complexidade, passariam a ser repassados ao município-sede de cada módulo, sendo o cálculo feito em valores per capita, de acordo com a população dos municípios da microrregião.

Os Estados, para se habilitarem na NOAS 01/01, passaram a ter que apresentar um Plano 
Estadual de Saúde, aprovado pelo respectivo Conselho Estadual de Saúde (CES). Neste Plano devia estar contido um Plano Diretor de Regionalização (PDR), uma Agenda de Compromissos Estadual e um Quadro de Metas, mediante o qual passaria a ser efetuado o acompanhamento dos Relatórios de Gestão. Esperava-se do gestor estadual a coordenação do processo de elaboração e de implementação das referências intermunicipais e os pactos de negociação para alocação dos recursos. Cabia-lhe também celebrar Termos de Compromisso para a Garantia de Acesso com os municípios-pólo correspondentes. A coordenação do sistema de referência e contra-referência intermunicipal passou a ser considerada uma atividade estratégica da gestão estadual. Suas habilitações no âmbito do sistema poderiam ser em duas formas de Gestão: Avançada do Sistema Estadual e Plena do Sistema Estadual.

O processo de implantação da NOAS O 110 I foi difícil, o que levou à sua reelaboração. Em algumas unidades da federação houve entraves decorrentes das dificuldades em estabelecer o comando único sobre os prestadores de serviços ao SUS, em assegurar a totalidade da gestão municipal nas sedes dos módulos assistenciais e em definir os mecanismos necessários à efetivação da gestão estadual para as referências intermunicipais. A revisão de seu texto, realizada por uma comissão formada por representantes do MS, CONASS e CONASEMS, gerou nova Norma Operacional de Assistência, a NOAS 01/02, aprovada em fevereiro de 2002.

A NOAS 01/02 manteve as diretrizes definidas pela anterior e procurou oferecer alternativas para a superação das dificuldades encontradas em sua operacionalização. Foram mantidos o PAB ampliado (PAB-A), as características das microrregiões de saúde e

CADERNOS GESTÃO PÚBLICA E CIDADANIA. V. 10. N. 46 - JAN/ABRIL 2005

10 A IMPLANTAÇÃO DO SUS E O PROCESSO DE DESCENTRALIZAÇÃO NA ÁREA DA SAÚDE ATÉ 2002

os mecanismos de financiamento para os procedimentos de maior complexidade e custo. As habilitações municipais passaram a denominar-se Gestão Plena da Assistência Básica Ampliada e Gestão Plena do Sistema Municipal.

Os municípios habilitados em Gestão Plena da Assistência Básica Ampliada passaram a receber um montante definido com base per capita para o financiamento das ações de atenção básica, definidas pela NOAS 01/02. Na Gestão Plena do Sistema Municipal, o município receberia o total dos recursos federais programados para o custeio da assistência em seu território. Os programas incluídos no valor do P AB-A permaneceram os mesmos da NOAS 01/01: o controle da tuberculose, a eliminação da hanseníase, o controle da hipertensão arterial, o controle do diabetes mellitus, a saúde da criança, a saúde da mulher e a saúde bucal.

Para os Estados, permaneceram as habilitações nas duas formas de Gestão já definidas: Avançada do Sistema Estadual e Plena do Sistema Estadual.

\section{Alguns resultados da descentralização até 2002}


A análise do processo de descentralização do SUS no período entre 1990 e 2002 evidencia que um primeiro e importante passo, foi a transferência, para os municípios, da gestão do sistema ambulatorial, por intermédio das Normas Operacionais Básicas. Também foi repassada para esta instância a responsabilidade pela atenção básica à saúde da população, com ênfase na promoção da saúde e na prevenção de agravos. Esta transferência tomou-se finalmente possível em função do financiamento estabelecido por meio de transferências fundo a fundo para os municípios, o $\mathrm{P}$ $\mathrm{AB}$, num segundo momento acompanhado pelo $\mathrm{P} A B-\mathrm{A}$.

Após a descentralização da atenção básica, o passo seguinte foi o estabelecimento de critérios para a regionalização da assistência. A experiência dos consórcios intermunicipais já vinha sendo realizada em diversos Estados, em alguns com mais sucesso do que em outros. Em função do sucesso não mais que relativo do modelo, esta alternativa de organização não se disseminou de forma homogênea por todo país (ABRUCIO, 1998). A solução encontrada para a regionalização foi a elaboração das Normas Operacionais de Assistência à Saúde.

CADERNOS GESTÃo PÚBLICA E CIDADANIA. V. ] O. N.46 - JAN/ABRIL 2005

A IMPLANTAÇÃO DO SUS E O PROCESSO DE DESCENTRALIZAÇÃO NA ÁREA DA SAÚDE A TÊ 2002

11

O processo de descentralização do sistema de saúde pode ser analisado em função de dois grandes momentos: uma primeira etapa de descentralização foi marcada pelas NOB 01/93 e NOB 01/96. Neste momento o principal foco da descentralização se deu sobre a atenção básica, a qual passou a ficar a cargo dos municípios e cuja principal estratégia foi a criação do PAB fixo e variável, com a definição do conjunto de procedimentos que implicariam na destinação do financiamento. Em um segundo momento, marcado pela NO AS 01/01 e NOAS 01/02, o foco principal do processo de descentralização passou a ser a regionalização do nível secundário da assistência. Em 2005 observa-se que todos os estados já estão em Gestão Plena.

Uma crítica comumente feita à NOAS 02/02 é o fato dela ser fortemente baseada nos estímulos financeiros para procedimentos diferentes, fazendo com que a oferta de serviços respondesse possivelmente mais à possibilidade de financiamento do que aos critérios epidemiológicos locais de necessidade. Uma das questões que o levantamento dos projetos encaminhados ao Programa Gestão Pública e Cidadania vai tentar responder, em artigo a ser proximamente publicado, é se de fato esta é uma realidade observada.

\section{Referências Bibliográficas}

ABRUCIO, Luiz F. (1998). Reforma do Estado e o Contexto Federativo Brasileiro, Pesquisa $n^{\circ}$ 12, Fundação Konrad Adenauer Stiftung, São Paulo. 
ARRETCHE, Marta .(2002). Relações Federativas nas políticas sociais, Educ. Soe., Campinas, v. 23, n. 80, setembro/2002, p. 25-48.

ARRETCHE, Marta \& MARQUES Eduardo (2002). Municipalização da saúde no Brasil: diferenças regionais, poder do voto e estratégias de governo. Ciência e Saúde Coletiva, 7 (3):455-479.

BONASSA, Elvis \& CAMPOS, Claudia (2001). Os programas e as formas de financiamento para os municípios. (mime o ) Ministério da Saúde. Brasília.

BRASIL. Manual para a Organização da Atenção Básica no Sistema Único de Saúde, Portaria n 3.925, de 13 de novembro de 1998 (Diário Oficial n² 220-E, de 17 de novembro de 1998, p. 814.

CADERNOS GESTÃO PÚBLICA E CIDADANIA. V. 10. N. 46 - JAN/ABRIL 2005

12 A IMPLANTAÇÃO DO SUS E O PROCESSO DE DESCENTRALIZAÇÃO NA ÁREA DA SAÚDE ATÉ 2002

BRASIL. Norma Operacional Básica 01/91. Nova Política de Financiamento do SUS. Resolução n 259 do Instituto Nacional de Assistência Médica da Previdência Social (Inamps), de 7 de janeiro de 1991. Diário Oficial de 10 de janeiro de 1991, Seção I, P. 641-4; Resolução nº 273 do Inamps, de 17 de julho de 1991. Diário Oficial de 18 de julho de 1991, p. 14.216-9 (reedição).

BRASIL. Norma Operacional Básica do Sistema Único de Saúde para 1992 (NOBSUS/92). Portaria da Secretaria Nacional de Assistência à saúde do Ministério da Saúde na 234, de 7 de fevereiro de 1992. Diário Oficial de 10 de fevereiro de 1992, p. 1.584-8.

BRASIL. Norma Operacional Básica 01/93. Estabelece normas e procedimentos reguladores do processo de descentralização das ações e serviços. Portaria do Gabinete do Ministro da Saúde n 545, de 20 de maio de 1993. Diário Oficial na 96, de 24 de maio de 1993, p. 6.961-5.

BRASIL. Norma Operacional Básica do Sistema Único de Saúde (NOB-SUS/96). Brasília: Ministério da Saúde, 1997. - Portaria do Gabinete do Ministro da Saúde nº 2.023 (Diário Oficial de 6 de novembro de 1996, p. 22.932-40); com alterações da Portaria do Gabinete do Ministro da Saúde $n^{\circ}$ 1.882, de 18 de dezembro de 1997 (Diário Oficial n² 247-E, de 22 de dezembro de 1997, p. 10-1), que estabelece o Piso de Atenção Básica (PAB) e sua composição. 
BRASIL. Norma Operacional de Assistência à Saúde (NOAS-SUS 01/01). Portaria do Ministério da Saúde n 95, de 26 de janeiro de 2001. Diário Oficial n 20-E, de 29 de janeiro de 2001.

BRASIL. Norma Operacional de Assistência à Saúde (NOAS-SUS 01/02). Portaria do Ministério da Saúde na 373, de 27 de fevereiro de 2002. Diário Oficial $n^{\circ}$ 40-E, de 28 de fevereiro de 2002.

COSTA, Luiz Renato L. da (2003). Os critérios de alocação de recursos financeiros no Sistema Único de Saúde: uma visão a partir das Normas Operacionais, 1991 a 2002.

Dissertação (Mestrado em Administração de Empresas) FGV-EAESP, São Paulo.

HEIMAN, Luisa S. et aI. (2000) Descentralização do Sistema Único de Saúde: trilhando a autonomia municipal. São Paulo: Sociedade Brasileira de Vigilância de Medicamentos.

CADERNOS GESTÃO PÚBLICA E CIDADANIA. V. 10, N.46 - JANI ABRIL 2005

A IMPLANTAÇÃO DO SUS E O PROCESSO DE DESCENTRALIZAÇÃO NA ÁREA DA SAÚDE ATÉ 2002

13

NEGRI, BaIjas (2002). A política de saúde no Brasil nos anos 1990: avanços e limites. ln: NEGRI, Barjas \& VIANA, Ana Luiza D'Âvila (org.) O Sistema Único de Saúde em dez anos de desafio. São Paulo: Sobravime; Cealag, 2002.

SANTOS, Gabriel Ferrato. Financiamento e investimento na saúde pública de 1995 a 2002. ln: NEGR1, Barjas \& VIANA, Ana Luiza D'Âvila (org.) O Sistema Único de Saúde em dez anos de desafio. São Paulo: Sobravime; Cealag, 2002.

SOUZA, Renilson Rehem. O sistema público de saúde brasileiro. ln: NEGR1, Barjas \& VIANA, Ana Luiza D' Â vila (org.) O Sistema Único de Saúde em dez anos de desafio. São Paulo: Sobravime; Cealag, 2002. 